Proc. Indian Acad. Sci. (Chem. Sci.), Vol. 96, No. 6, April 1986, pp. 499-508.

(C) Printed in India.

\title{
Nonlinear conduction and electrical switching in one-dimensional conductors
}

\author{
S V SUBRAMANYAM* and HEMAMALINI NAIK \\ Department of Physies, Indian Institute of Science, Bangalore 560012, India
}

\begin{abstract}
Many one-dimensional conductors show pronounced nonlinear electrical conduction. Some of them show very interesting electrical switching from a low conducting state to a high conducting state. Such electrical switching is often associated with memory. These are discussed with particular emphasis on charge transfer complexes TMBine-YCNQ, TMPD-TCNQ, $\mathrm{Cs}_{2}(\mathrm{TCNQ})_{3}, \mathrm{TEA}-(\mathrm{TCNQ})_{2}$ and 0 -tolidine-iodine.
\end{abstract}

Keywords. One-dimensional conductors; charge transfer complexes; electrical switching; memory phenomena; nonlinear conduction; charge density waves; high pressure phenomena.

\section{Introduction}

Many charge transfer complexes exhibit interesting properties such as a deviation from ohmic behaviour and switching from a low conducting state to a high conducting state (Subramanyam and Hemamalini Naik 1985). Such behaviour has been observed previously in a wide variety of systems ranging from amorphous chalcogenides like $\mathrm{Te}_{48} \mathrm{As}_{30} \mathrm{Ge}_{10} \mathrm{Si}_{12}$ (Csillag 1973), insulating films of mylar, organic thin films of aromatic hydrocarbons like tetracene, anthracene (Elsharkawi and Kao 1977), polycrystalline thin films of $\mathrm{Cu}$-7,7,8,8-tetracyanoquinodimethane (TCNQ) (Potember et al 1979) and chalcogenides like $\mathrm{NbSe}_{3}$ (Zettl and Gruner 1982).

\subsection{Non-ohmic conduction}

A deviation from ohmic behaviour at high driving fields has been reported in a number of compounds such as tetrathiofulvalene (TIF)-rCNQ (Gunning and Heeger 1978),

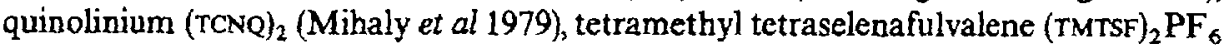
(Chaikin et al 1980) $\mathrm{NbSe}_{3}$ and $\mathrm{TaS}_{3}$ (Fleming and Grimes 1979; Miller et al 1983) and $(\mathrm{CH})_{x}$ (Epstein et al 1980). Pronounced deviation from ohmicity under applied high pressures at high driving fields has been observed in some organic charge transfer complexes like $\mathbf{N}, \mathbf{N}, \mathbf{N}^{\prime}, \mathbf{N}^{\prime}$-tetramethyl-p-phenylenediamene TMPD-TCNQ, $N, N_{,} \mathbf{N}^{\prime}, \mathbf{N}^{\prime}$ tetramethyl benzidine TMBine-TCNQ (Bandyopadhyay and Subramanyam 1979), $\mathrm{Cs}_{2}(\mathrm{TCNQ})_{3}$, triethylammonium TEA-(TCNQ) $)_{2}$ (Hemamalini Naik and Subramanyam 1984, 1986b; Hemamalini Naik et al 1983) and o-tolidine-iodine (Hemamalini Naik and Subramanyam 1983b, 1986a; Hemamalini Naik et al 1982).

A number of theories have been proposed to fit the experimental data, none of which satisfactorily explains the data. In most of the cases, non-linearity was attributed to the depinning of the charge density wave (CDW) by an applied field. A pinned CDW

\footnotetext{
* To whom all correspondence should be addressed.
} 
corresponds to a situation wherein it is held in a potential varying non-linearity with the displacement from a pinned site. Such systems exhibit nonlinear response to any external perturbation. The nonlinear current-voltage (I-v) characteristics in a pinned CDW system can arise from various phenomena such as solitons. The depinning of $\mathrm{CDW}$ by zener tunnelling has also been considered (Miller et al 1983). The other mechanisms considered are space charge limited currents (Farges et al 1972), phonon assisted hopping through random barriers, impurity pinning (Lee and Rice 1979) and sliding CDW (Frohlich 1954).

\subsection{Electrical switching and memory phenomena}

It is known that the characteristic electrical properties of many materials undergo drastic changes at high fields in excess of $10^{6} \mathrm{~V} \mathrm{~cm}^{-1}$. In insulators, such high fields lead to a destructive breakdown while in some amorphous materiais, such effects can be nondestructive. In these cases, the physical processes after breakdown are quite different from those before and are of considerable interest and have been exhaustively studied (Turnbull and Bagley 1975; Klein et al 1971; Le Comber and Mort 1973). Nondestructive breakdown is closely related to switching. Both have similar developments leading to a change in the material from a low conducting $O F F$ state to a high conducting $O N$ state with $\sigma_{\mathrm{ON}} / \sigma_{\mathrm{OFF}} \sim 10^{2}-10^{4}$.

Switching is a result of application of an electric field higher than a threshold onto the specimen. Once the specimen has switched to the $O N$ state, it behaves in one of the two ways to an applied bias: it either returns to the OFF state once the bias is removed in which case it is called threshold switching, or it remains in the $O N$ state even after the applied bias is removed in which case, it is called memory switching. The different properties of the two states find a number of applications (Fritzsche 1973). To study what initiates switching, the dependence of the switching field on parameters such as electrode materials, sample thickness, temperature and pressure must be considered.

The I-v characteristics and electrical switching, as a function of high hydrostatic pressure (upto $7 \mathrm{GPa}$ ), yield interesting results. A number of charge transfer complexes (1DC) such as TMBine-TNCQ, TMPD-TCNQ, $\mathrm{Cs}_{2}(\mathrm{TCNQ})_{3}, \mathrm{TEA}(\mathrm{TCNQ})_{2}$ and $o$-tolidine-iodine have been subjected to such studies.

Various mechanisms of thermal and electronic origin have been considered to explain the switching phenomena. This phenomena is not very well understoad. Under pressure, various factors have to be taken into consideration. The pressure dependence of the switching field and activation energy are not very well understood. A comprehensive review of our experimental results in these areas is given here.

\section{Experiments}

Reversible and rapid bistable switching has been observed in polycrystalline samples of $\mathrm{Cu}$-TCNO sandwiched between two metal electrodes with switching field less than $3 \times 10^{3} \mathrm{~V} \mathrm{~cm}^{-1}$ (Potember et al 1979). The device acts either as a threshold switch or as a memory switch depending on the strength and duration of the applied field. The switching behaviour can be directly related to the reduction potential of the acceptor.

Anthracene thin films sandwiched between metal strips also show thereshold switching (Elsharkawi and Kao 1977). After several switching cycles, the switching 
changed to a memory type. Switching was observed only in samples less than $5 \mu \mathrm{m}$ thickness.

In $\mathrm{NbSe}_{3}$, threshold switching shows up in direct $I-V$ traces and leads to a hysteresis in increasing and decreasing driving currents (Zettl and Gruner 1982) at $T=26.5 \mathrm{~K}$. With increasing temperature, the hysteresis becomes progressively smaller disappearing at $T=38 \mathrm{~K}$ where nonlinear conduction begins.

The $I-V$ characteristics have been studied under pressure up to $7 \mathrm{GPa}$ of some charge transfer complexes (figures 1-4). The characteristics show a strong deviation from the ohmic behaviour before switching to the $O N$ state. The nonlinearity is more pronounced with increasing pressures. The switching current also increases with an increase in pressure.while the switching field decreases (figure 5). The switching field is typically $10^{3} \mathrm{~V} \mathrm{~cm}^{-1}$ and $\sigma_{O N} / \sigma_{O F F} \sim 10^{2}-10^{4}$. The driving force in all these cases is a current source.

In TMBine-TCNQ, the $O N$ state can be driven back to the $O F F$ state by the application of an a.c. pulse of a suitable frequency and duration. In the case of TMPD-TCNQ, the OFF state is restored by warming the sample to about $50^{\circ} \mathrm{C}$.

\section{Theory}

\subsection{Non-ohmic conduction}

Non-ohmic conduction has been exhaustively studied in the linear chain compounds $\mathrm{NbSe}_{3}$ (Monceau et al 1976; Ong and Monceau 1977). Early measurements gave the

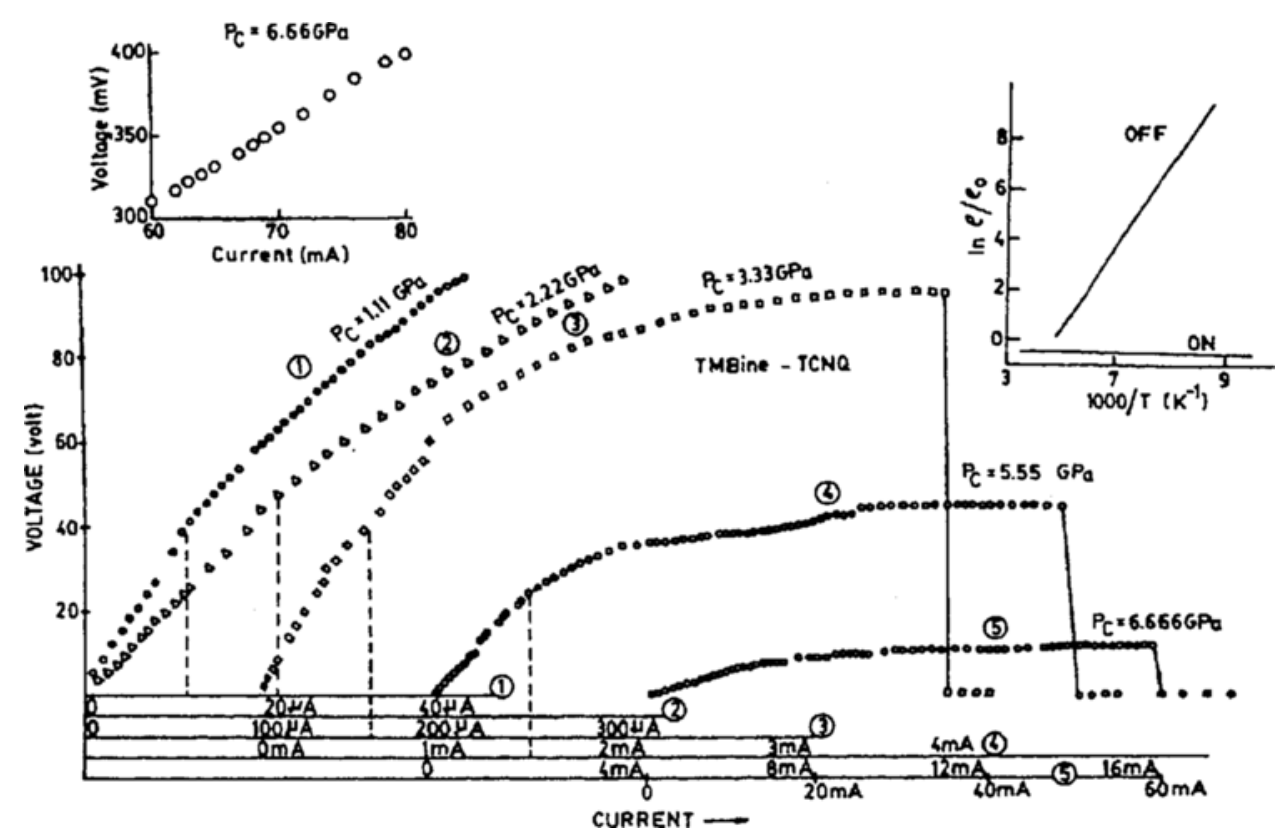

Figure 1. Current-voltage characteristics of TMBine-TCNQ at high pressures. (inset shows current-voltage characteristics after switching and in $\rho / \rho_{0}$ vs $1000 / T$ for the two states). 


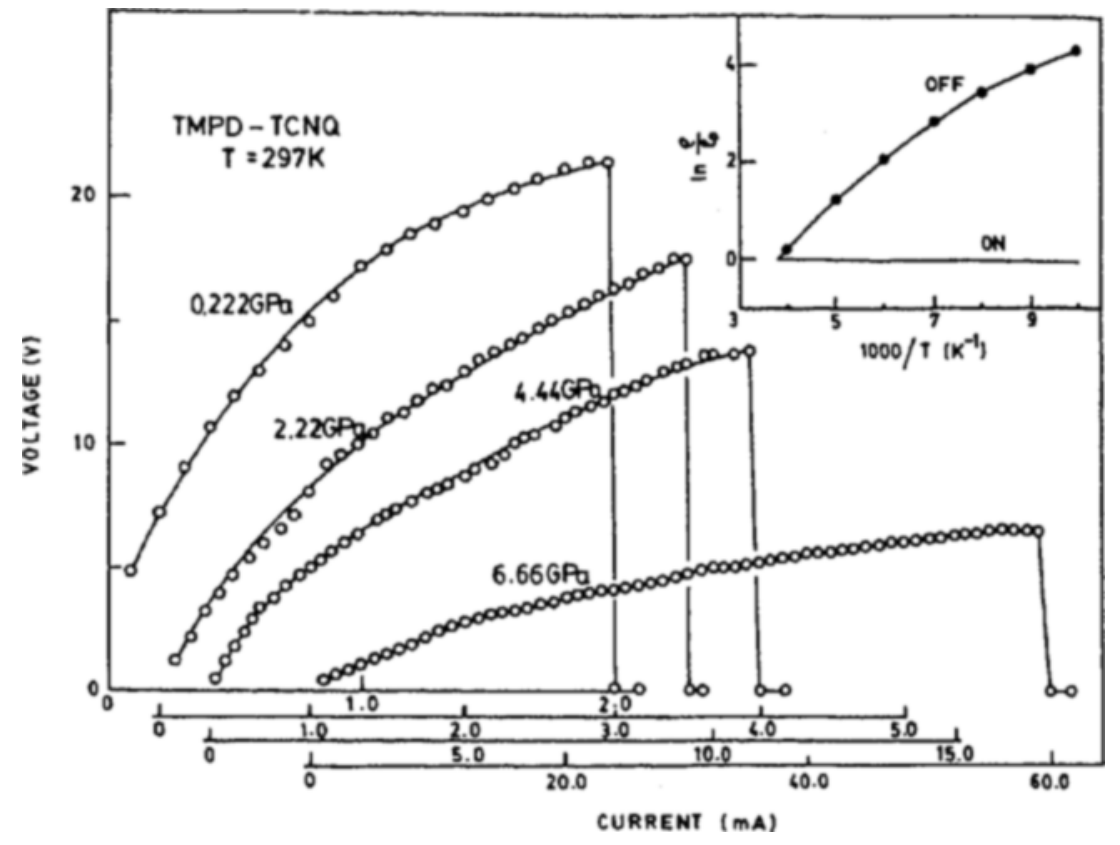

Figure 2. Current-voltage characteristics of TMPD-TCNQ at high pressures. (inset shows $1000 / T$ for $O N$ and $O F F$ states).

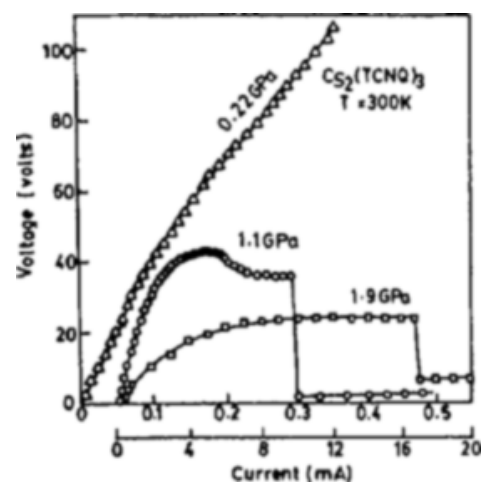

Figure 3. I- $V$ characteristics of $\mathrm{Cs}_{2}(\mathrm{TCNO})_{3}$ at different pressures.

value for the field dependent conductivity as

$$
\sigma(F)=\sigma_{a}+\sigma_{b} \exp \left(-F_{0} / F\right) .
$$

The second term on the right hand side is due to the sliding charge density waves. This expression for the field dependent conductivity suggests a zener tunnelling across a pinning gap. Later Brill et al (1981) showed that the data could be fitted approximately by a modified tunnelling formula where $F$ is replaced by $F-F_{T}\left(F>F_{T}\right), F_{T}$ being the threshold field. A revised scaling relation between field and frequency gives good agreement between a.c. and d.c. coupling effects providing strong evidence in favour of 


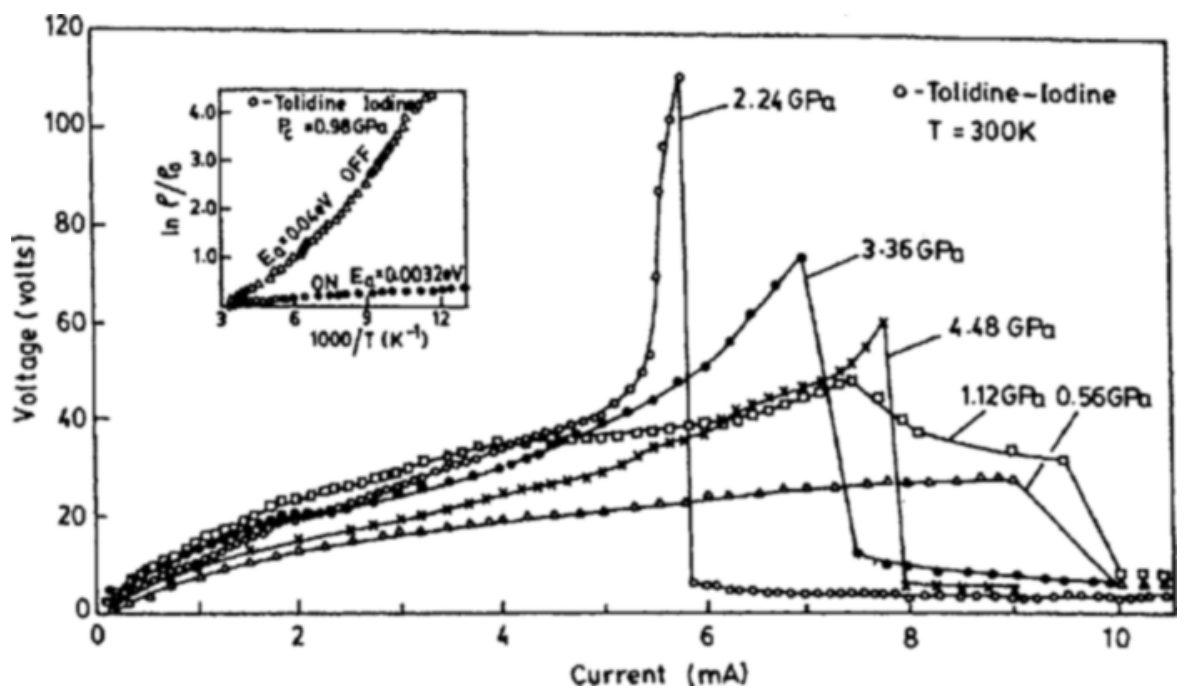

Figure 4. $L-V$ characteristics of $o$-tolidine-iodine at different pressures.

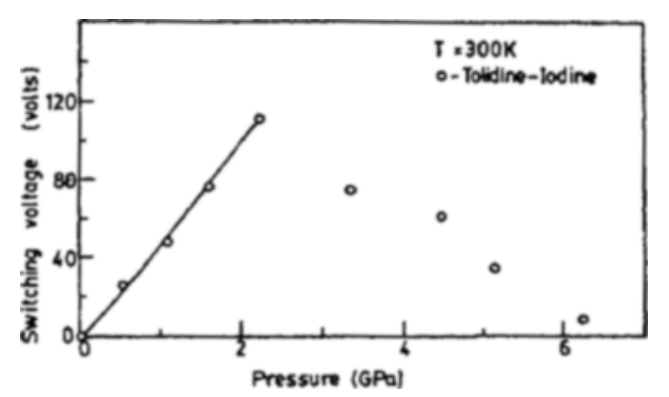

Figure 5. Switching voltage as a function of pressure for o-tolidine-iodine.

the tunnelling interpretation of the CDW dipinning (Miller et al 1983). A revised relation of the above tunnelling equation is

$$
I_{\text {d.c }}=G_{a} F+G_{b}\left(F-F_{T}\right) \exp \left(-F_{0} / F\right),
$$

where $F_{0}$ is the characteristic field for zener tunnelling. The linear term represents the ohmic conductivity and the second term arises due to the CDW.

Alternatively, the $I-V$ characteristics can also be fitted to an equation of the type

$$
I(E)=A\left(1-e^{-B E}\right)\left(e^{C E+D E^{2}}\right) .
$$

\subsection{Electrical switching}

Two possible theories can be thought of to explain the switching phenomena based on the thermal and electronic origin. The former may arise due to high power dissipation in the sample while the latter is due to the increase in the number or mobility of the 
electrons. Switching could also be the result of electrode injection or consequences of non-ohmic conduction. In the latter case, one can invoke two models to explain the switching phenomena: depinning of the CDW and tunnelling through the coulomb barrier of charged impurities. Switching observed in $\mathrm{NbSe}_{3}$ is associated with the CDW state. Dielectric breakdown (O'Dwyer 1973) has also been considered as a possible mechanism. In the presence of an electric field, the electron gains energy, some energy is lost due to collisions with the phonons. If the energy gained exceeds the energy lost, for some electric field, then the energy of the electron can increase indefinitely resulting in breakdown of the system.

Some of the other mechanisms invoked to explain the switching mechanism are the field induced solid state reversible electrochemical reaction associated with the charge transfer salts as in the case of Cu-TCNQ (Potember et al 1981; Kamitsos et al 1982)

$$
\left(\mathrm{Cu}^{+} \mathrm{TCNQ}^{-}\right)_{n} \rightleftharpoons \mathrm{Cu}_{x}^{0}+\mathrm{TCNQ}_{x}^{0}+\left(\mathrm{Cu}^{+} \mathrm{TCNQ}^{-}\right)_{n-x} .
$$

This case has been verified using various spectroscopic techniques.

It was suggested that the switching observed in anthracene thin films was due to the formation of stacks of charge transfer complexes across samples between the electrodes leading to easy charge transfer. This can happen via donor-acceptor interactions which are retained even after the removal of the applied field and broken when an a.c. pulse is applied; although the data available are insufficient to identify it.

\section{Discussion}

\subsection{Non-ohmic conduction}

The non-ohmic part of the $I-V$ characteristics of TMPD-TCNQ and TMBine-TCNQ have been fitted to an equation of the type

$$
I(F)=A[1-\exp (-B F)] \exp \left(C F+D F^{2}\right)
$$

and the fit is found to be satisfactory.

In the case of $\mathrm{TEA}-(\mathrm{TCNQ})_{2}, \mathrm{Cs}_{2}(\mathrm{TCNQ})_{3}$ and $o$-tolidine-iodine, it has been fitted to an equation of the type

$$
\sigma(F)=\sigma_{a}+\sigma_{b} \exp \left(-F_{0} / F\right)
$$

Figure 6 gives a plot of $\ln \left[\sigma(F)-\sigma_{a}\right]$ as a function of $1 / F$ of TEA $(\mathrm{TCNQ})_{2}, \mathrm{Cs}_{2}(\mathrm{TCNQ})_{3}$ and $o$-tolidine-iodine respectively. As seen from the figure, the equation gives a good line on fit. Hence, in all these cases, the non-ohmic conductivity has been attributed to the zener tunnelling type of $\mathrm{CDW}$. Though the formation of $\mathrm{CDW}$ is questionable at room temperature, the applied pressure plays an important role (Hemamalini Naik and Subramanyam 1984, 1986; Hemamalini Naik et al 1983a).

Nonlinear transport has been observed in TTF-TCNQ and related compounds KCP and $\mathrm{NbSe}_{3}$ (Cohen and Heeger 1977). For the TrF compounds, Lee et al (1974) suggested a hopping type motion of the pinned $\mathrm{CDW}$ condensate and a resulting field dependent conduction. Cohen and Heeger (1977) suggested a conduction of the type $\sigma=\sigma_{0}\left(1+\beta E^{2}\right)$ where $\beta$ takes into account the defects and impurities. The unambiguous proof for the intrinsic nature of the nonlinear transport came from microwave harmonic mixing. 


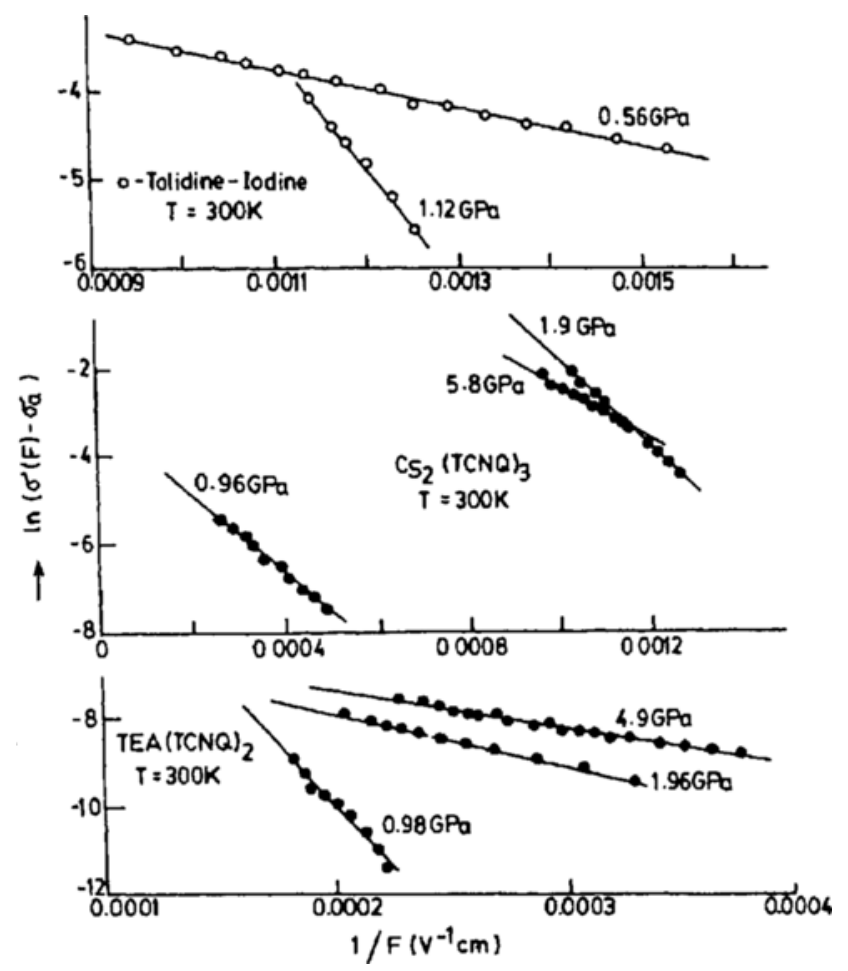

Figure 6. Variation of $\ln \left[\sigma(F)-\sigma_{a}\right]$ of the charge transfer complexes with the inverse of field.

\subsection{Electrical switching}

Electrical switching has been most extensively studied in amorphous chalcogenides. Here it has been suggested that when the heat generated is greater than the heat lost, an instability occurs whereby the current and the self-heated region collapse to a filament establishing a new stationary state rapidly within a switching time $<10^{-10} \mu \mathrm{sec}$ (The $O N$ state dynamic resistance is $1-10 \mathrm{ohm}$ ). Switching characteristics are obtained as solutions to the heat transport and charge conservation equations subject to proper boundary conditions, device geometry, external circuitry and material properties. As long as the current exceeds a minimum or holding current, the system is in its $O N$ state. When the current is reduced, it returns to the $O F F$ state within $1 \mu \mathrm{sec}$.

When the switching has been observed under pressure, the various mechanisms considered are dielectric breakdown, zener tunnelling, thermal breakdown etc.

4.2a Dielectric breakdown: Above a critical field, if the electron gains more energy than it loses to the lattice, then its energy increases considerably resulting in the breakdown of the system by the relation

$$
P>\hbar W_{D}^{2} \text {, }
$$

where $P$ is the energy gained by every electron, $\hbar$ the Planck's constant and $W$ the Debye 
frequency. $P$ depends on the switching power, the sample geometry and the number of electrons/cc.

Calculations done for typical cases show that this mechanism is not responsible for switching in the present experiments.

4.2b Zener tunnelling: The application of pressure reduces the band gap increasing the probability of zener tunnelling from the valence band to the conduction band. An increase in the probability is also enhanced as a result of switching when the activation energy is considerably reduced. We have the probability for such a process

$$
P_{v-\mathrm{c}}=\exp \left[-(2 / 3 e F)\left(2 m^{*} / h^{2}\right)^{\frac{1}{2}} \Delta^{3 / 2}\right],
$$

where $F$ is the switching field and $\Delta$ the activation energy after switching. When substitution is made for various pressures, the probability is found to be negligible. These calculations are not exact, but do indicate that zener tunnelling is not the cause of switching.

4.2c Thermal breakdown: Thermal breakdown can occur when the conductivity has a strong dependence on temperature. The application of an electric field produces Joule heating which increases the temperature which in turn increases the conductivity and hence the current. When the joule heat generated by the current flow is greater than the heat lost by conduction, an instability occurs which drives the system into a new state (Miller et al 1983). In all the cases where the charge transfer complexes are studied under pressure, they are firmly embedded in the steatite pressure transmitting medium between the tungsten carbide anvils. Moreover, the $O N$ state is not very activated. The total amount of heat supplied to the sample at the time of switching is less than 0.2 watts. Measurements of the temperature of the sample do not show any appreciable increase in temperature during the switching experiment. This amount of heat is distributed over the entire sample and dissipated to its surroundings. Hence thermal runaway has been ruled out as a possible mechanism.

4.2d Electrochemical process: That electrical switching is an electrochemical reaction of the type observed in $\mathrm{Cu}$-TCNQ has been ruled out as the temperature dependence of the conductivity in the two states is quite different from that observed in Cu-TCNQ. Also the complexes are of the donor-acceptor type and not of the metal-salt type.

\section{Memory phenomenon}

When the $O N$ state is maintained even after the driving current is removed, it is called memory switching. Whenever a marked change in properties accompanies a bistable switching, it has device potential, as a memory for information storage. In an electrical memory, the stored information is read out and erased electrically. The two conducting states are both stable at zero electrical bias. For optimum instability, $\rho_{O F F} / \rho_{O N} \sim 10^{5}$ or less. To produce the memory $O N$ state, a critical 'set time' has to be exceeded. Memory phenomenon has been extensively studied in chalcogenide oxides. The nature and the state of memory in charge transfer complexes are not fully understood. But this has high physical significance and technological applications. 


\section{Conclusions}

Detailed experiments on the d.c. conductivity of organic quasi one-dimensional conductors at high pressures have clearly demonstrated pronounced nonlinear conduction and electrical switching. The nonlinear conduction is explained on the zener tunnelling model. Electrical switching is associated with memory effects. Dielectric breakdown, thermal breakdown and zener tunnelling are not responsible for switching. The electrical switching observed is attributed to some collective electronic excitations in the complex.

\section{Acknowledgements}

The authors are thankful to DST New Delhi and ISRO-IISc Space Technology Cell, Bangalore, for financial support. They are thankful to their colleagues A K Bandyopadhyay, S Chatterjee, Raghu and others for active participation in the work. They are also highly thankful to Prof. C N R Rao for his interest and appreciation of the work.

\section{References}

Brill J W, Ong N P, Eckert J C, Savage J W, Khanna S K and Somoano R B 1981 Phys. Rev. B23 1517 Bandyopadhyay A K and Subramanyam S V 1979 Nucl. Phys. Solid State Phys. (India) C22 1874

Chaikin P M, Gruner G, Engber E M and Greene R L 1980 Phys. Rev. Lett. 451874

Cohen M J and Heeger A J 1977 Phys. Rev. B16 688

Csillag A 1973 in Electronic and structural properties of amorphous semiconductors (eds) P G Le Camber and J Mort (Orlando: Academic Press) p. 319

Elsharkawi A R and Kao K C 1977 J. Phys. Chem. Solids 3895

Epstein A J, Gibson H W, Chaikin P M, Clark W G and Gruner G 1980 Phys. Rev. Lett. 451730

Farges J P, Bran A and Guttman 1972 J. Phys. Chem. Solids 331723

Fleming R M and Grimes C C 1979 Phys. Rev. Lett. 421423

Fritzsche H 1973 in Electronic and structural properties of amorphous semiconductors (eds) P G Le Camber and J Mort (Orlando: Academic Press) p. 279

Frohlich H 1954 Proc. R. Soc. London A233 296

Gunning W J and Heeger A J 1978 Solid State Commun. 27843

Hemamalini Naik, Chatterjee S and Subramanyam S V 1982 Nucl. Phys. Solid State Phys. (India) C25 342

Hemamalini Naik, Chatterjee S and Subramanyam S V 1983a J. Inst. Soc. (India) 13307

Hemamalini Naik and Subramanyam S V 1984 Nucl. Phys. Solid State Phys. (India) C27 193

Hemamalini Naik and Subramanyam S V 1983b Proc. Symp. amorphous materials, Bombay (Bombay: D A E) p. 269

Hemamalini Naik and Subramanyam S V 1986a Pramana (J. Phys.) 2661

Hemamalini Naik and Subramanyam S V $1986 \mathrm{~b}$ Synth. Met. (communicated)

Kamitsos E I, Tzimis S H and Risin W M Jr 1982 Solid State Commun. 42561

Klein N, Tannhauses D S and Pollak M 1971 Conduction in low mobility materials (London: Taylor and Francis)

Le Camber P G and Mort J (eds) 1973 Electronic and structural properties of amorphous semiconductors (Orlando: Academic Press)

Lee P A and Rice T M 1979 Phys. Rev. B19 3970

Lee P A, Rice T M and Anderson P W 1974 Solid State Commun. 14703

Mihaly G, Janosty A, Kurti A, Ferro J and Gruner G 1979 Phys. Status Solidi. B94 287

Miller J H Jr, Richard J, Tucker J R and Bardeen J 1983 Phys. Rev. Lett. 511592

Mortensen K, Thewak M L K, Tomikieuncz Y, Clarke T C and Street G B 1980 Phys. Rev. Lett. 45490 
Monceau P, Ong N P, Portis A M, Meerschant A and Rouxes J 1976 Phys. Rev. Lett. 37602 O'Dwyer J J 1973 The theory of electrical conduction and breakdown in solid dielectrics (Oxford: Clarendon Press)

Ong N P and Monceau P 1977 Phys. Rev. B16 3443

Potember R S, Poehler T O and Cowan D D 1979 Appl. Phys. Lett. 34405

Potember R S, Poehler T O, Cowan D O and Bloch A N 1980 in Physics and chemistry of low dimensional solids (ed.) L Alcacer (Dordrecht: D Reidel) p. 419

Potember R S, Poehler T O, Cowan D O, Brant P, Carter F L and Block A N 1981 Chem. Scri. 17219

Subramanyam $S$ V and Hemamalini Naik 1985 in The metallic and non-metallic states of matter: An important facet of the chemistry and physics of the condensed state (eds) P P Edwards and C N R Rao (London and Philadelphia: Taylor and Francis)

Subramanyam S V 1981 in Preparation and characterisation of materials (eds) J M Havig and C N R Rao (London: Academic Press) p. 367

Turnbull D and Bagley B G 1975 Treatise on solid state chemistry (ed.) N B Hannay (New York: Plenum Press) p. 513

Zettl A and Gruner G 1982 Phys. Rev. B26 2298 